\title{
Cohort Profile of the NEIGE Study in Tokamachi City, Japan
}

\author{
Yugo Shobugawa $^{1}$, Hiroshi Murayama ${ }^{2}$, Takeo Fujiwara ${ }^{3}$, and Shigeru Inoue ${ }^{4}$ \\ ${ }^{1}$ Division of International Health, Niigata University Graduate School of Medical and Dental Sciences, Niigata, Japan \\ ${ }^{2}$ Institute of Gerontology, The University of Tokyo, Tokyo, Japan \\ ${ }^{3}$ Department of Global Health Promotion, Tokyo Medical and Dental University, Tokyo, Japan \\ ${ }^{4}$ Department of Preventive Medicine and Public Health, Tokyo Medical University, Tokyo, Japan
}

Received March 5, 2019; accepted May 7, 2019; released online May 25, 2019

\begin{abstract}
Background: Studies have shown that rural residents face disadvantages concerning medical access and socio-economic conditions. However, the social determinants of health among older people in rural areas are still unclear. The Neuron to Environmental Impact across Generations (NEIGE) study investigated the social determinants of health among older rural adults. Methods: A survey was conducted among the older residents of Tokamachi City, Japan. We randomly selected study participants $(N=1,346)$ from four stratified groups by age (65-74 and 75-84 years) and residential area (Tokamachi center [downtown] and Matsunoyama [mountain]). The survey collected data on socio-economic status, lifestyle, health, and neighborhood environment. Blood and urine sampling were also conducted, and physical activity was assessed. Magnetic resonance brain imaging (MRI) and Apo-E gene were also examined in the analysis.

Results: In total, 527 people participated in the NEIGE study (participation rate: $39.2 \%$ ). The average age of the participants was 73.5 (standard deviation, 5.6) years, and $47.3 \%$ were male. No differences in demographics were found between downtown and mountain residents, except for educational attainment, which was lower among mountain residents. Lifestyles were similar, except for the higher percentage of everyday drinkers (33.3-35.3\%) in the mountain area. Concerning physical health, muscle mass, grip strength, and measured physical activity were significantly higher among mountain residents. However, gait speed and balance were better among downtown residents.

Conclusion: The findings of the NEIGE study will help elucidate the social determinants of health in older rural adults in Japan, and emphasize the different outcomes between downtown and mountain areas.
\end{abstract}

Key words: older adults; rural health; social determinants of health; cohort profile

Copyright @ 2019 Yugo Shobugawa et al. This is an open access article distributed under the terms of Creative Commons Attribution License, which permits unrestricted use, distribution, and reproduction in any medium, provided the original author and source are credited.

\section{INTRODUCTION}

Japan represents one of the most rapidly aging societies in the world, with this phenomenon more remarkable in rural areas. ${ }^{1}$ Older adults living in rural areas have limited medical resources, so they face disadvantages related to health., ${ }^{2,3}$ Lower health literacy and lower socio-economic status among older rural residents might also cause poor health outcomes. ${ }^{4,5}$

Universal health coverage is one of the most important issues promoted by the World Health Organization (WHO) and a key aspect of the United Nations' Sustainable Development Goals (SDGs). ${ }^{6}$ Thus, the promotion of health among older adults residing in rural areas is essential.

The social determinants of health have been well investigated. However, some factors affect the health of older adults in urban and rural areas in different ways. For instance, social capital generally affect physical and psychological health positively. ${ }^{7,8}$ However, some studies have shown a null or opposite association in rural areas; for example, one study showed that particularized trust did not influence health in rural areas, ${ }^{9}$ while another one showed that bridging social capital was positively associated with depressive mood among women in rural settings. ${ }^{9,10}$ Since geographic, cultural, and historical contexts differ between urban and rural areas, the social determinants of health should be investigated and compared between rural and urban conditions. Furthermore, agricultural work has been determined to be an important social determinant of health for older rural adults. Several studies showed that gardening, which is included in agriculture, promotes mental health. ${ }^{1-14}$ However, there few studies have investigated the association between agricultural work and health for older adults.

Therefore, we launched a cohort study titled the Neuron to Environmental Impact across Generation (NEIGE) study in 2017 to investigate the social determinants of health among older rural residents. The present report partially describes the results of the NEIGE study's baseline data.

\section{METHODS}

Study design, setting, and participants

The study population comprised individuals aged 65 through 84 
years living in Tokamachi City, Niigata Prefecture, Japan. Tokamachi City is an agricultural city located 150 kilometers north of Tokyo. As of July 2017, the population was 54,515 (26,560 males and 27,955 females), with 20,089 people aged 65 years or older (proportion, 36.9\%). The area of the city is approximately 590 square kilometers, with a low population density ( 87.8 persons per square kilometer). From the target age (65-84 years old) population (15,792 people) in the whole city, residents of the downtown and mountain areas of the city were examined, and their characteristics were compared to analyze their differences between social determinants of older health. The center of the city located downtown is the most populated area; conversely, the mountain area, set apart from the center, was selected as a representative of the least populated area. The distance between these two areas is $24 \mathrm{~km}$. Tokamachi City receives heavy snowfall, with $9,978 \mathrm{~cm}$ (downtown) and $19,710 \mathrm{~cm}$ (mountain) in the winter of 2017 . The name of the study, "NEIGE," is also the French word for "snow."

The target population in the selected downtown and mountain areas consisted of the 1,524 residents of Tokamachi, aged 65 to 84 years as of July 1, 2017. People with long-term care certification and those residing in nursing homes were excluded from the analysis. From the target population, 1,346 older adults were sampled. Then, invitations to participate in the study were sent to the sampled population via mail. We recruited participants using the stratified random sampling method based on the resident register. Equal numbers of participants were recruited for each age group (ie, 65-69, 70-74, 75-79, and 80-84) and also stratified by sex. As there is a large difference in environment and lifestyle between downtown residents and mountain residents, we also stratified the participants by residential area (downtown vs mountain). The sample size was calculated using the following formula:

$$
n=p(1-p)\left(\frac{z}{E}\right)^{2}
$$

$n=$ sample size required in each group

$z=1.96$ for $95 \%$ CI

$E=$ desired margin of error $=0.05$

$p=$ proportion of functional declining defined as long-term care insurance certification. (15\% [roughly estimated] of the LTCI certification percentage gathered from the national data)

$$
\begin{aligned}
& n=0.15(1-0.15)\left(\frac{1.96}{0.05}\right)^{2} \\
& n=196
\end{aligned}
$$

Therefore, the minimum required sample size was 196 in each stratified group.

A total of 527 people participated in the NEIGE study (Figure 1). There were no monetary or other incentives for participation. Before the survey, we informed participants of the research purpose and method.

\section{Baseline survey}

The baseline survey was conducted in the mountain area in September and in the downtown area in October 2017. Participants completed the survey in person. The main items surveyed are summarized in Table 1.

Demographics: Age, sex, living arrangement, and socioeconomic status. Socio-economic status includes educational attainment, income, and current employment.

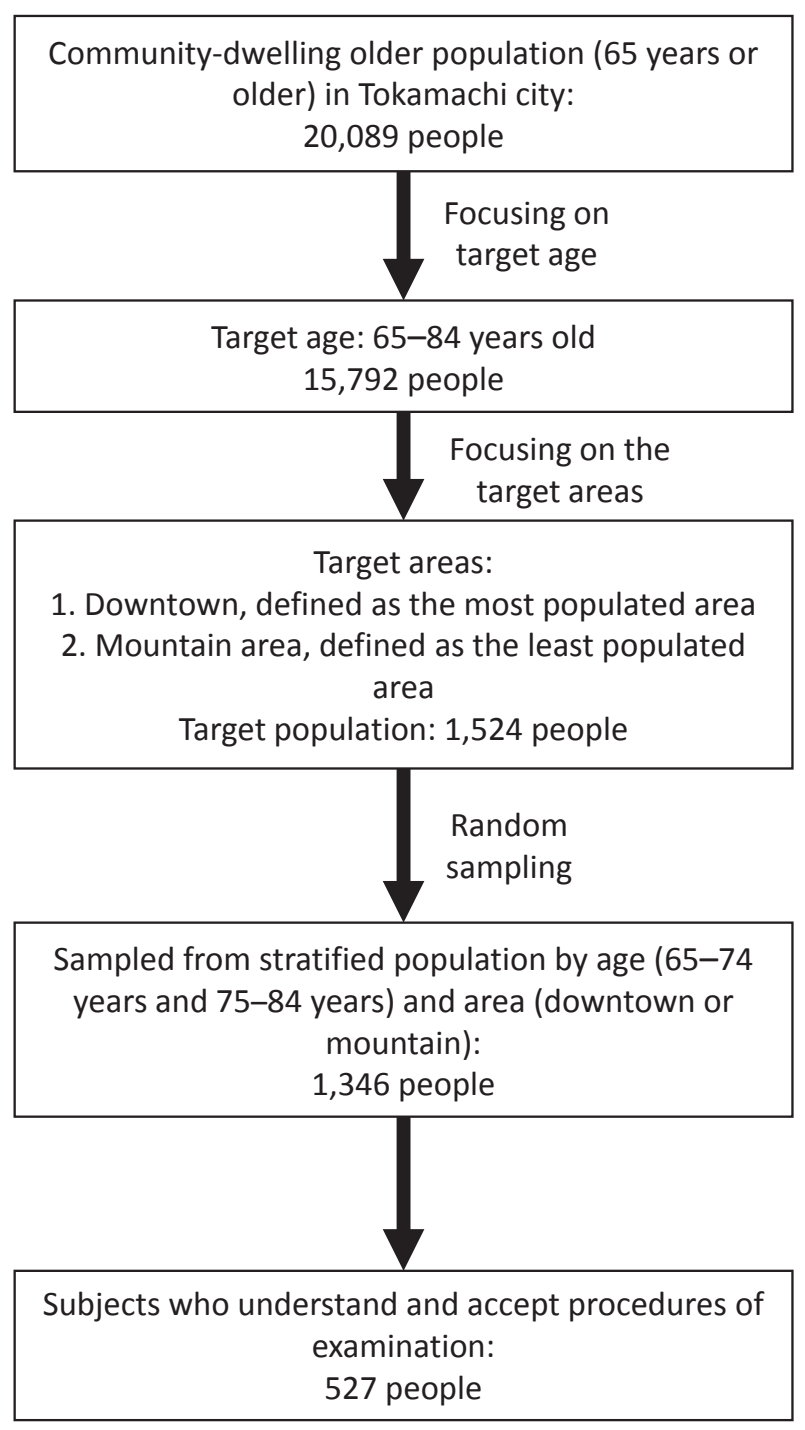

Figure 1. Flow diagram of the study participants.

Lifestyle: Agricultural work, smoking habit, alcohol consumption, sleep duration, and physical activity were measured using an accelerometer HJA-750C Active style Pro (Omron Healthcare, Kyoto, Japan). The geographical range of daily activities was captured using the Global Positioning System (GPS). To measure physical activity, a cutoff based on metabolic equivalents (METs) was used; $\leq 1.5$ METs for sedentary behavior and $\geq 3.0$ METs for moderate-to-vigorous physical activity. Accelerometer and GPS were prepared for auto recording; no handlings were needed for the participants.

Nutritional function: Dietary habits, including food intake variety, were assessed using the questionnaire (10 kinds of foods [eg, meats, vegetables, and milk $]^{15,16}$ ). Biomarkers of nutrition were tested through blood samples, including evaluation of serum albumin, total cholesterol, and blood hemoglobin. Urinary sodium chloride was measured through laboratory testing to estimate salt intake per day.

Medical characteristics: History of physician-diagnosed diseases (past or/and current) and number of prescribed medications were obtained by interviewers (medical doctors or nurses).

Body composition: Body mass index (BMI), abdominal circumference, fat mass, and muscle mass. BMI was calculated 
Table 1. Summary of baseline survey items in the 2017 NEIGE study

\begin{tabular}{|c|c|}
\hline Items & Description \\
\hline Demographics & $\begin{array}{l}\text { Age, sex, family members, marital status, } \\
\text { equivalized income, educational attainment, } \\
\text { employment status }\end{array}$ \\
\hline Lifestyle & $\begin{array}{l}\text { Agricultural work, smoking, alcohol } \\
\text { consumption, sleep duration, physical } \\
\text { activity measured by accelerometer, } \\
\text { geographical range of daily activity } \\
\text { measured by GPS }\end{array}$ \\
\hline Nutritional function & $\begin{array}{l}\text { Frequency of meat/fish consumption, } \\
\text { frequency of vegetable/fruit consumption, } \\
\text { food intake variety, biomarkers of nutrition, } \\
\text { urinary sodium chloride }\end{array}$ \\
\hline Medical characteristics & $\begin{array}{l}\text { History of physician-diagnosed diseases } \\
\text { (past or/and current), number of prescribed } \\
\text { medications }\end{array}$ \\
\hline Body composition & $\begin{array}{l}\text { Body mass index, abdominal circumference, } \\
\text { body composition, fat mass, muscle mass }\end{array}$ \\
\hline Cardiovascular function & Blood pressure, heart rate \\
\hline Physical function & $\begin{array}{l}\text { Grip strength } \\
\text { Gait speed } \\
\text { Balance }\end{array}$ \\
\hline Psychological function & Geriatric depression scale \\
\hline Cognitive function & $\begin{array}{l}\text { MMSE-J, analysis of para-hippocampal } \\
\text { atrophy using MRI }\end{array}$ \\
\hline Activities of daily living & $\begin{array}{l}\text { Basic activities of daily living } \\
\text { Higher-order competence of independence } \\
\text { (TMIG-IC) }\end{array}$ \\
\hline Neighborhood environment & Physical environment, social capital \\
\hline
\end{tabular}

from measured body height and body weight. Fat mass and muscle mass were estimated from bioelectrical impedance analysis using a body composition analyzer MC-780A (TANITA Corporation, Tokyo, Japan). All measurement was conducted by the research staff (no self-measurement).

Cardiovascular function: Blood pressure and heart rate were measured using a blood pressure monitor UA-1020 (A \& D Medical, Saitama, Japan). The measurement was conducted by health staff (medical doctor or nurse).

Physical function: Grip strength, 5-meter usual gait speed, and standing time on one foot with eyes open (maximum: 60 seconds). Grip strength was measured twice in each hand using a dynamometer. Gait speed was calculated as time (seconds) taken for a 5-meter walk. Balance was evaluated based on standing time on one foot. The measurement was conducted by trained staff.

Psychological function: The Geriatric Depression Scale (GDS) short-form (15 items) $)^{17-20}$ and self-rated health.

Cognitive function: The Mini-Mental State Examination Japanese version (MMSE-J ${ }^{21}$ and analysis of para-hippocampal atrophy using Magnetic resonance brain image. ${ }^{22}$ VSRAD (Voxel-based Specific Regional analysis system for Alzheimer's Disease) was applied to study para-hippocampal atrophy. ${ }^{23}$ MMSE-J was evaluated by trained staff.

Activities of daily living (ADL): Basic ADL and higherlevel functional capacity. The Tokyo Metropolitan Institute of Gerontology Index of Competence, which consists of three subscales (instrumental self-maintenance, intellectual activity, and social role) were used. ${ }^{24}$
Neighborhood environment: neighborhood living environment and neighborhood social capital, including civic participation, general trust, and attachment to neighbors. To measure social capital, a method from the previous study was partially applied. ${ }^{25}$

We also investigated genetic factors including polymorphism of the Apo-E gene and sense of hearing and smell. Remaining blood samples were kept for further analyses.

\section{Follow-up outcome surveys}

We will follow-up on three survey items: health outcomes, lifespan, and long-term care insurance (LTCI) certification. To measure individual changes in health outcomes, we will conduct a follow-up survey every 3 years in the same manner as the baseline survey. To follow survival time and LTCI certification since the baseline survey, we will confirm information from Tokamachi City annually.

\section{Ethical considerations}

The study protocol was reviewed and approved by the Ethics Committee of Niigata University on November 25, 2016 (approval number: 2666). Written informed consent was obtained from all study participants.

\section{Statistical analysis}

To describe the baseline characteristics of the study participants, we compared the main measures among the participants according to age (65-74 vs 75-84 years old) and residential area (downtown vs mountain) using the chi-square test for nominal variables, the Kruskal-Wallis test for ordinal variables, and $t$-test for continuous variables. Statistical significance was set as a $P$ value of less than 0.05 on a two-tailed test. All the analyses were performed using STATA 15 (Stata Corp, College Station, TX, USA).

\section{RESULTS}

Table 2 displays results concerning the demographic, life style, physical and mental health status. The agricultural working rate was significantly higher among mountain residents in both age groups (both $P<0.001$ ). The marriage rate was lower among downtown residents aged 65-74 $(P=0.03)$ than among mountain residents (Table 3), while educational attainment was higher among downtown residents than mountain residents in both age groups. Alcohol consumption was significantly higher in the mountain area in the $75-84$ group $(P=0.012)$. Objectively measured physical activity showed that sedentary time was longer among downtown residents (452.0 [standard deviation $\{\mathrm{SD}\}$, 122.2] vs 412.0 [SD, 140.4], $P=0.009)$, and moderate-tovigorous physical activity time was shorter (57.2 [SD, 36.3] vs 71.8 [SD, 45.9], $P=0.002$ ) among downtown residents aged 65-74. For body composition, muscle mass was significantly higher among the mountain residents and fat mass was higher among downtown residents. Blood pressure did not differ between downtown and mountain residents. Urinary sodium chloride, which can be used to estimate salt intake, was significantly higher among downtown residents in both age groups. Grip strength was stronger among mountain residents, while gait speed was faster among downtown residents of both age groups. Finally, balance was better among downtown residents in both age groups. The results of the MRI are not shown because validation of the obtained data is ongoing. 
Cohort Profile of the NEIGE Study Tokamachi Japan

Table 2. Summary of the main items surveyed according to sex and age group in the NEIGE study

\begin{tabular}{|c|c|c|c|c|c|c|}
\hline & & $\begin{array}{l}\text { Total } \\
N=527\end{array}$ & $\begin{array}{l}\text { Male } \\
65-74 \text { years } \\
N=149\end{array}$ & $\begin{array}{l}75-84 \text { years } \\
N=100\end{array}$ & $\begin{array}{l}\text { Female } \\
65-74 \text { years } \\
N=102\end{array}$ & $\begin{array}{l}75-84 \text { years } \\
N=104\end{array}$ \\
\hline \multicolumn{7}{|l|}{ Demographics } \\
\hline Age & Mean (SD) & $73.5(5.6)$ & $69.5(2.7)$ & $79.5(2.9)$ & $69.3(2.8)$ & $79.1(2.7)$ \\
\hline Sex & Men, $\%$ & 47.3 & - & - & - & - \\
\hline Family members & Living alone, $\%$ & 8.9 & 4.0 & 4.0 & 9.4 & 18.5 \\
\hline Marital status & Married, \% & 80.5 & 94.0 & 91.0 & 79.3 & 56.3 \\
\hline Equivalized annual income, JPY & 2.00 million or above & 54.2 & 66.5 & 46.0 & 56.6 & 42.9 \\
\hline Educational attainment, years & 10 years or longer, $\%$ & 61.7 & 83.9 & 56.0 & 62.9 & 36.9 \\
\hline Employment status & Currently employed, $\%$ & 41.2 & 53.0 & 38.0 & 46.5 & 21.9 \\
\hline \multicolumn{7}{|l|}{ Lifestyle } \\
\hline Agricultural work & Currently working, $\%$ & 58.4 & 67.1 & 56.0 & 52.8 & 57.1 \\
\hline Smoking & Current smoker, $\%$ & 8.9 & 18.1 & 13.0 & 3.8 & 0.8 \\
\hline Alcohol consumption & Every day or sometimes, $\%$ & 53.2 & 81.9 & 73.0 & 38.4 & 20.1 \\
\hline Sleeping duration & Mean (SD) & $7.7(1.2)$ & $7.7(1.1)$ & $8.5(1.2)$ & $7.1(1.0)$ & $7.6(1.1)$ \\
\hline \multirow[t]{2}{*}{ Physical activity } & Sedentary time, $\min /$ day & $445.4(129.7)$ & $456.8(141.9)$ & $486.1(136.7)$ & $408.8(120.1)$ & $447.5(107.0)$ \\
\hline & $\begin{array}{l}\text { Moderate-to-vigorous physical activity time, } \\
\mathrm{min} / \text { day }\end{array}$ & $52.3(39.9)$ & $65.9(43.4)$ & $38.6(33.4)$ & $63.3(40.7)$ & $31.4(24.2)$ \\
\hline \multicolumn{7}{|l|}{ Nutritional function } \\
\hline Frequency of meat/fish consumption & Everyday, \% & 58.4 & 54.4 & 57.0 & 54.1 & 70.6 \\
\hline Frequency of vegetable/fruit consumption & Everyday, \% & 89.0 & 91.3 & 86.0 & 86.2 & 92.4 \\
\hline Food intake variety & Mean (SD) & $5.5(2.2)$ & $5.2(2.2)$ & $5.3(2.5)$ & $5.5(2.2)$ & $6.1(1.9)$ \\
\hline \multirow[t]{3}{*}{ Biomarkers of nutrition } & Serum albumin, g/dL Mean (SD) & $4.4(0.3)$ & $4.4(0.3)$ & $4.3(0.3)$ & $4.5(0.3)$ & $4.4(0.3)$ \\
\hline & Total cholesterol, mg/dL Mean (SD) & $203(33)$ & $199(33)$ & $190(34)$ & $214(30)$ & $207(33)$ \\
\hline & Blood hemoglobin, g/dL Mean (SD) & $13.6(1.4)$ & $14.6(1.2)$ & $14.0(1.3)$ & $13.2(1.0)$ & $12.7(1.2)$ \\
\hline Urinary sodium chloride, g per day & Mean $(\mathrm{SD})$ & $9.7(2.1)$ & $9.9(2.1)$ & $9.9(2.3)$ & $9.7(2.0)$ & $9.5(2.1)$ \\
\hline \multicolumn{7}{|l|}{ Body composition } \\
\hline Body mass index, $\mathrm{kg} / \mathrm{cm}^{2}$ & Mean (SD) & $22.1(3.0)$ & $22.6(2.5)$ & $22.8(2.6)$ & $21.7(3.2)$ & $21.5(3.3)$ \\
\hline Abdominal circumference, $\mathrm{cm}$ & Mean (SD) & $83.0(9.2)$ & $84.9(7.5)$ & $86.9(8.1)$ & $80.7(9.8)$ & $80.5(9.5)$ \\
\hline Fat mass, $\%$ & Mean (SD) & $24.1(8.3)$ & $18.1(5.6)$ & $21.2(6.2)$ & $28.3(7.7)$ & $28.3(7.9)$ \\
\hline Muscle mass, $\mathrm{kg}$ & Mean (SD) & $39.5(7.7)$ & $47.8(4.3)$ & $44.5(5.1)$ & $34.0(2.7)$ & $32.2(2.9)$ \\
\hline \multicolumn{7}{|l|}{ Cardiovascular function } \\
\hline Systolic blood pressure, $\mathrm{mm} \mathrm{Hg}$ & Mean (SD) & $138(19)$ & $138(17)$ & $141(18)$ & $135(20)$ & $140(20)$ \\
\hline Diastolic blood pressure, $\mathrm{mm} \mathrm{Hg}$ & Mean (SD) & $82(11)$ & $85(11)$ & $81(11)$ & $82(10)$ & $81(11)$ \\
\hline Heart rate, beats $/ \mathrm{min}$ & Mean (SD) & $70(12)$ & $69(11)$ & $69(11)$ & $70(10)$ & $73(13)$ \\
\hline \multicolumn{7}{|l|}{ Physical function } \\
\hline Grip strength, kg & Mean (SD) & $30.1(8.2)$ & $38.7(5.9)$ & $33.6(6.5)$ & $25.3(3.7)$ & $22.6(3.7)$ \\
\hline Gait speed, $\mathrm{m} / \mathrm{s}$ & Mean (SD) & $1.3(0.3)$ & $1.3(0.2)$ & $1.2(0.3)$ & $1.4(0.2)$ & $1.2(0.3)$ \\
\hline Standing time on 1 foot with eyes open, seconds & Mean (SD) & $34.5(23.3)$ & $42.7(21.8)$ & $25.7(22.3)$ & $41.7(21.4)$ & $25.1(21.9)$ \\
\hline \multicolumn{7}{|l|}{ Psychological function } \\
\hline Geriatric Depression Scale & Mean (SD) & $2.8(2.7)$ & $2.4(2.3)$ & $2.5(2.3)$ & $2.9(2.9)$ & $3.4(3.1)$ \\
\hline \multicolumn{7}{|l|}{ Cognitive function } \\
\hline Mini-Mental Status Examination Japanese version & Mean (SD) & $27.0(2.6)$ & $26.6(2.9)$ & $27.2(2.6)$ & $26.7(2.6)$ & $27.6(2.2)$ \\
\hline \multicolumn{7}{|l|}{ Activities of daily living (ADL) } \\
\hline Instrumental self-maintenance (range: $0-5$ ) & Full points, $\%$ & 53.3 & 29.5 & 27.0 & 81.8 & 67.2 \\
\hline Intellectual activity (range: $0-4$ ) & Full points, $\%$ & 68.1 & 76.5 & 69.0 & 63.5 & 63 \\
\hline Social role (range: $0-4$ ) & Full points, \% & 61.3 & 53.0 & 50.0 & 71.7 & 67.2 \\
\hline \multicolumn{7}{|l|}{ Neighborhood environment } \\
\hline \multicolumn{7}{|l|}{ Physical environment: } \\
\hline park or walking road & Yes, $\%$ & 63.2 & 58.4 & 70.0 & 61.6 & 65.6 \\
\hline Grocery & Yes, \% & 66.7 & 59.7 & 75.0 & 69.2 & 65.3 \\
\hline \multicolumn{7}{|l|}{ Social capital } \\
\hline \multirow[t]{3}{*}{ Civic participation } & None, $\%$ & 25.2 & 17.5 & 38.0 & 20.8 & 30.3 \\
\hline & One, $\%$ & 29.4 & 36.2 & 24.0 & 33.3 & 20.2 \\
\hline & Two or more, $\%$ & 45.4 & 46.3 & 38.0 & 45.9 & 49.6 \\
\hline General trust & Yes, \% & 80.1 & 84.6 & 83.0 & 73.6 & 80.7 \\
\hline Attachment to neighbor & Yes, \% & 88.4 & 91.3 & 91.0 & 83.0 & 89.9 \\
\hline
\end{tabular}


Shobugawa Y, et al.

Table 3. Summary of the main measures surveyed divided by residential area and age group in the NEIGE study

\begin{tabular}{|c|c|c|c|c|c|c|c|c|}
\hline & & \multirow{2}{*}{$\begin{array}{l}\text { Total } \\
N=527\end{array}$} & \multicolumn{2}{|c|}{ Urban (downtown) } & \multicolumn{2}{|c|}{ Rural (mountain) } & \multicolumn{2}{|c|}{$\begin{array}{l}P \text { between downtown } \\
\text { and mountain }\end{array}$} \\
\hline & & & $\begin{array}{l}65-74 \text { years } \\
N=155\end{array}$ & $\begin{array}{l}75-84 \text { years } \\
N=126\end{array}$ & $\begin{array}{l}65-74 \text { years } \\
N=153\end{array}$ & $\begin{array}{l}75-84 \text { years } \\
N=93\end{array}$ & $\begin{array}{l}\text { Among } \\
65-74 \text { years }\end{array}$ & $\begin{array}{l}\text { Among } \\
75-84 \text { years }\end{array}$ \\
\hline \multicolumn{9}{|l|}{ Demographics } \\
\hline Age & Mean (SD) & $73.5(5.6)$ & $69.5(2.8)$ & $79.2(2.8)$ & $69.2(2.7)$ & $79.3(2.8)$ & ns & ns \\
\hline Sex & Male, $\%$ & 47.3 & 41.9 & 43.7 & 54.9 & 48.4 & 0.023 & ns \\
\hline Family members & Living alone, $\%$ & 8.9 & 6.5 & 9.5 & 7.2 & 15 & ns & ns \\
\hline Marital status & Married, \% & 80.5 & 81.3 & 70.6 & 91.5 & 74.2 & 0.03 & ns \\
\hline Equivalized income, JPY & 2.00 million or above & 54.2 & 63.3 & 52.4 & 59.5 & 33.4 & $\mathrm{~ns}$ & ns \\
\hline Educational attainment, years & 10 years or longer, $\%$ & 61.7 & 81.9 & 54.8 & 64.1 & 33.4 & 0.002 & 0.007 \\
\hline Employment status & Currently employed, $\%$ & 41.2 & 54.2 & 27.8 & 45.1 & 31.2 & $\mathrm{~ns}$ & 0.045 \\
\hline \multicolumn{9}{|l|}{ Lifestyle } \\
\hline Agricultural work & Currently working, $\%$ & 58.4 & 27.1 & 34.1 & 92.8 & 87.1 & $<0.001$ & $<0.001$ \\
\hline Smoking & Current smoker, $\%$ & 8.9 & 9.7 & 6.4 & 11.8 & 6.5 & ns & ns \\
\hline Alcohol consumption & Every day or sometimes, $\%$ & 53.2 & 62.0 & 42.9 & 56.9 & 46.2 & ns & 0.012 \\
\hline Sleep duration & Hours, Mean (SD) & $7.7(1.2)$ & $7.4(1.1)$ & $7.9(1.2)$ & $7.4(1.1)$ & $8.2(1.2)$ & ns & ns \\
\hline \multirow[t]{2}{*}{ Physical activity } & Sedentary time, min/day Mean (SD) & $445.4(129.7)$ & $452.0(122.2)$ & $477.8(127.2)$ & $412.0(140.4)$ & $448.5(114.9)$ & 0.009 & $\mathrm{~ns}$ \\
\hline & $\begin{array}{l}\text { Moderate-to-vigorous physical } \\
\text { activity time, min/day Mean (SD) }\end{array}$ & $52.3(39.9)$ & $57.2(36.3)$ & $34.2(30.0)$ & $71.8(45.9)$ & $35.2(27.4)$ & 0.002 & ns \\
\hline \multicolumn{9}{|l|}{ Nutritional function } \\
\hline Frequency of meat/fish consumption & Everyday, \% & 58.4 & 58.1 & 65.1 & 50.3 & 63.4 & ns & ns \\
\hline $\begin{array}{l}\text { Frequency of vegetable/fruit } \\
\text { consumption }\end{array}$ & Everyday, \% & 89.0 & 86.5 & 90.5 & 90.9 & 88.2 & $\mathrm{~ns}$ & ns \\
\hline Food intake variety & Mean (SD) & $5.5(2.2)$ & $5.3(2.3)$ & $5.8(2.2)$ & $5.5(2.1)$ & $5.7(2.3)$ & ns & ns \\
\hline \multirow[t]{3}{*}{ Biomarkers of nutrition } & Serum albumin, g/dL Mean (SD) & $4.4(0.3)$ & $4.4(0.3)$ & $4.4(0.3)$ & $4.5(0.3)$ & $4.4(0.3)$ & ns & ns \\
\hline & Total cholesterol, mg/dL Mean (SD) & $203(33)$ & $210.6(33.7)$ & $200.9(37.1)$ & $201.9(30.4)$ & $197.0(30.4)$ & 0.018 & ns \\
\hline & Blood hemoglobin, g/dL Mean (SD) & $13.6(1.4)$ & $13.8(1.3)$ & $13.2(1.4)$ & $13.2(1.4)$ & $13.4(1.3)$ & ns & ns \\
\hline Urinary sodium chloride, g per day & Mean (SD) & $9.7(2.1)$ & $10.1(2.0)$ & $9.9(2.0)$ & $9.4(2.0)$ & $9.3(2.3)$ & 0.003 & 0.038 \\
\hline \multicolumn{9}{|l|}{ Body composition } \\
\hline Body mass index, $\mathrm{kg} / \mathrm{cm}^{2}$ & Mean (SD) & $22.1(3.0)$ & $22.3(2.9)$ & $22.3(2.9)$ & $22.0(3.0)$ & $21.9(3.3)$ & ns & ns \\
\hline Abdominal circumference, $\mathrm{cm}$ & Mean (SD) & $83.0(9.2)$ & $82.0(9.4)$ & $83.2(9.6)$ & $83.5(8.5)$ & $83.7(9.4)$ & ns & ns \\
\hline Fat mass, $\%$ & Mean (SD) & $24.1(8.3)$ & $25.1(8.5)$ & $26.0(7.1)$ & $21.6(8.2)$ & $23.7(9.0)$ & $<0.001$ & 0.04 \\
\hline Muscle mass, $\mathrm{kg}$ & Mean (SD) & $39.5(7.7)$ & $70.8(8.1)$ & $69.5(9.2)$ & $74.2(7.8)$ & $71.5(11.3)$ & $<0.001$ & ns \\
\hline \multicolumn{9}{|l|}{ Cardiovascular function } \\
\hline Systolic blood pressure, $\mathrm{mm} \mathrm{Hg}$ & Mean (SD) & $138(19)$ & $136.1(16.6)$ & $139.1(18.7)$ & $137.1(20.8)$ & $142.1(19.9)$ & ns & ns \\
\hline Diastolic blood pressure, $\mathrm{mm} \mathrm{Hg}$ & Mean (SD) & $82(11)$ & $83.7(10.1)$ & $79.8(10.6)$ & $83.2(11.2)$ & $82.1(10.9)$ & ns & $\mathrm{ns}$ \\
\hline Heart rate, beats/min & Mean (SD) & $70(12)$ & $70.1(11.5)$ & $71.4(11.5)$ & $68.7(10.1)$ & $70.7(13.6)$ & ns & $\mathrm{ns}$ \\
\hline \multicolumn{9}{|l|}{ Physical function } \\
\hline Grip strength, kg & Mean (SD) & $30.1(8.2)$ & $30.2(7.9)$ & $27.0(7.7)$ & $33.5(8.3)$ & $28.4(7.4)$ & 0.001 & $\mathrm{~ns}$ \\
\hline Gait speed, $\mathrm{m} / \mathrm{s}$ & Mean (SD) & $1.3(0.3)$ & $1.4(0.2)$ & $1.3(0.3)$ & $1.3(0.2)$ & $1.1(0.2)$ & $<0.001$ & $<0.001$ \\
\hline Balance, seconds & Mean (SD) & $34.5(23.3)$ & $48.4(18.8)$ & $28.2(22.2)$ & $35.9(22.5)$ & $21.3(21.3)$ & $<0.001$ & 0.025 \\
\hline \multicolumn{9}{|l|}{ Psychological function } \\
\hline Geriatric Depression Scale & Mean (SD) & $2.8(2.7)$ & $2.9(2.9)$ & $3.3(3.0)$ & $2.4(2.3)$ & $2.6(2.4)$ & ns & ns \\
\hline \multicolumn{9}{|l|}{ Cognitive function } \\
\hline $\begin{array}{l}\text { Mini-Mental Status Examination } \\
\text { Japanese version }\end{array}$ & Mean (SD) & $27.0(2.6)$ & $2.9(2.9)$ & $3.3(3.0)$ & $2.4(2.3)$ & $2.6(2.4)$ & ns & ns \\
\hline \multicolumn{9}{|l|}{ Activities of daily living (ADL) } \\
\hline $\begin{array}{l}\text { Instrumental self-maintenance } \\
\text { (range: } 0-5 \text { ) }\end{array}$ & full points, $\%$ & 53.3 & 56.8 & 47.6 & 56.2 & 50.5 & ns & 0.038 \\
\hline Intellectual activity (range: 0-4) & full points, $\%$ & 68.1 & 76.8 & 68.3 & 62.8 & 62.4 & 0.06 & ns \\
\hline Social role (range: $0-4$ ) & full points, $\%$ & 61.3 & 60.7 & 59.5 & 64.7 & 59.1 & $\mathrm{~ns}$ & ns \\
\hline \multicolumn{9}{|l|}{ Neighborhood environment } \\
\hline \multicolumn{9}{|l|}{ Physical environment } \\
\hline park or walking road & Yes, $\%$ & 63.2 & 77.4 & 75.4 & 42.5 & 57.0 & $<0.001$ & 0.004 \\
\hline Grocery & Yes, $\%$ & 66.7 & 87.1 & 81.0 & 41.8 & 54.4 & $<0.001$ & $<0.001$ \\
\hline Social capital & & & & & & & & \\
\hline Civic participation & None, $\%$ & 25.2 & 15.5 & 27.0 & 22.9 & 43.0 & & \\
\hline & One, $\%$ & 29.4 & 33.6 & 22.2 & 36.0 & 21.5 & & \\
\hline & Two or more, $\%$ & 45.4 & 51.0 & 50.8 & 41.2 & 35.5 & ns & 0.032 \\
\hline General trust & Yes, $\%$ & 80.1 & 79.4 & 79.4 & 78.4 & 85.0 & ns & ns \\
\hline Attachment to neighbor & Yes, $\%$ & 88.4 & 85.8 & 88.9 & 88.2 & 92.5 & ns & ns \\
\hline
\end{tabular}




\section{DISCUSSION}

The NEIGE study is a cohort study, and this report partially describes data at the baseline survey. Our findings showed that muscle mass and grip strength were higher among the mountain residents. Although these residents face disadvantages concerning access to medical care and socio-economic status (including educational attainment and household income), which can cause lower instrumental ADL, cognitive impairment, or even higher mortality rates, ${ }^{26}$ rural mountain life and agricultural work can often improve residents' health. Our baseline data indicates that older mountain residents generally had better physical capacities than their downtown counterparts. However, the results of the gait speed and balance tests were poorer for mountain residents.

Compared to the participants of other cohort studies, such as the Kashiwa Cohort Study and NILS-LSA, hand grip strength was found to be stronger in older adults from Tokamachi. ${ }^{27,28}$ However, the gait speed of the participants in the other studies was similar to or faster than the participants from Tokamachi. The burden of agricultural work might decrease scores for several physical functions because of curved posture, lumbago, and knee pain. Percent fat mass was higher for participants, specifically older age participants (75 or older), in Tokamachi than for those in NILS-LSA. ${ }^{28}$ This difference might be caused because of the maintenance of muscle mass due to the continuance of agricultural work even after the standard retirement age (65 years old) in rural areas. However, this requires further investigation. In our study, lower GDS scores were presented among older adults in the mountain area, but were statistically not significant. This finding might support previous studies showing that gardening reduces stress and anxiety. ${ }^{11-14}$

The strengths of the NEIGE study include the following. First, the study is a comprehensive investigation of physical and mental functions, social factors, spatial factors, genetic factors, and objective measurements, such as accelerometer and magnetic resonance imaging. Such multiple factors can be utilized to elucidate significant causes of functional decline and frailty. Second, geographical records and objectively measured physical activity data were used. These data make it possible to evaluate physical activity in more detail using objective rather than subjective measurements. Third, face-to-face interviews were used to complete the baseline surveys; this is a more effective method for completing the dataset as compared to questionnaire surveys, which may have missing data. Fourth, we will follow-up on the participants through face-to-face interviews and collect data on lifespan and LTCI certification. Older rural residents rarely move to other areas; thus, we do not anticipate much difficulty following-up on the study participants. Follow-up data can elucidate longitudinally social determinants of health and mortality.

The limitations of the study are as follows. First, the participants were randomly selected within the target area, but the participants were volunteers who could come to the survey location. Thus, the people who participated in the survey were possibly healthier than average for this population. Second, the effect of agricultural work on mental health needs to be further investigated.

In conclusion, the NEIGE study was launched in 2017 to evaluate the social determinants of health among older Japanese residents from rural areas. An additional follow-up survey will be conducted 3 years after the baseline survey. Information on lifespan and LCTI certification will be also collected. The NEIGE study will contribute to designing health policies that promote healthy and active lifestyles among older adults in Japan.

\section{ACKNOWLEDGEMENTS}

This study was funded by a grant from 'the Policy Research Institute, Ministry of Agriculture, Forestry and Fisheries', JSPS KAKENHI Grant Number 16H03249, 17K19794, 18K10829, and 19H03910 and a grant from 'the Pfizer Health Research Foundation'. We would like to thank Tokamachi City for their help in organizing the survey. We would specifically like to thank Yasuko Kodama for her help with the arrangements in Tokamachi City and Tomoko Manabe for her excellent secretarial work.

Conflicts of interest: None declared.

\section{REFERENCES}

1. United Nations Department of Social and Economic Affairs. World Population Ageing 2017. Highlights. http://www.un.org/en/ development/desa/population/publications/pdf/ageing/WPA2017. Highlights.pdf.

2. Sullivan MW, Camacho FT, Mills AM, Modesitt SC. Missing information in statewide and national cancer databases: correlation with health risk factors, geographic disparities, and outcomes. Gynecol Oncol. 2019;152(1):119-126.

3. Vanasse A, Courteau J, Cohen AA, Orzanco MG, Drouin C. Ruralurban disparities in the management and health issues of chronic diseases in Quebec (Canada) in the early 2000s. Rural Remote Health. 2010;10(4):1548.

4. Golboni F, Nadrian H, Najafi S, Shirzadi S, Mahmoodi H. Urbanrural differences in health literacy and its determinants in Iran: a community-based study. Aust J Rural Health. 2018;26(2):98-105.

5. Parker EJ, Mills H, Spencer AJ, Mejia GC, Roberts-Thomson KF, Jamieson LM. Oral health impact among rural-dwelling indigenous adults in South Australia. J Health Care Poor Underserved. 2016; 27(1)(Suppl):207-219.

6. World Health Organization and the International Bank for Reconstruction and Development/The World Bank 2017. Tracking universal health coverage: 2017 global monitoring report. https://apps. who.int/iris/bitstream/handle/10665/259817/9789241513555eng.pdf;jsessionid=5EE8E5A5D39053F862262D2C946DCA9A? sequence $=1$. Published December, 2017. Accessed 15.04.2019.

7. Kawachi I, Kennedy BP, Glass R. Social capital and self-rated health: a contextual analysis. Am J Public Health. 1999;89:11871193.

8. Kawachi I, Berkman LF. Social ties and mental health. J Urban Health. 2001;78:458-467.

9. Murayama H, Nofuji Y, Matsuo E, et al. Are neighborhood bonding and bridging social capital protective against depressive mood in old age? A multilevel analysis in Japan. Soc Sci Med. 2015;124:171179.

10. Sato Y, Aida J, Tsuboya T, et al. Generalized and particularized trust for health between urban and rural residents in Japan: a cohort study from the JAGES project. Soc Sci Med. 2018;202:43-53.

11. Barnes KL, Bendixsen CG. "When this breaks down, it's black gold": race and gender in agricultural health and safety. J Agromed. 2017;22:56-65.

12. Van Den Berg AE, Custers MH. Gardening promotes neuroendocrine and affective restoration from stress. J Health Psychol. 2011;16:3-11.

13. Mackay GJ, Neill JT. The effect of "green exercise" on state anxiety and the role of exercise duration, intensity, and greenness: a quasiexperimental study. Psychol Sport Exerc. 2010;11:238-245.

14. Bowler DE, Buyung-Ali LM, Knight TM, Pullin AS. A systematic review of evidence for the added benefits to health of exposure to 
natural environments. BMC Public Health. 2010;10:456.

15. Kumagai S, Watanabe S, Shibata $H$, et al. Effects of dietary variety on declines in high-level functional capacity in elderly people living in a community. Nihon Koshu Eisei Zasshi. 2003;50:1117-1124.

16. Kwon J, Suzuki T, Kumagai S, Shinkai S, Yukawa H. Risk factors for dietary variety decline among Japanese elderly in a rural community: a 8-year follow-up study from TMIG-LISA. Eur J Clin Nutr. 2006;60:305-311.

17. Almeida OP, Almeida SA. Short versions of the geriatric depression scale: a study of their validity for the diagnosis of a major depressive episode according to ICD-10 and DSM-IV. Int J Geriatr Psychiatry. 1999;14(10):858-865.

18. D'Ath P, Katona P, Mullan E, Evans S, Katona C. Screening, detection and management of depression in elderly primary care attenders. I: the acceptability and performance of the 15 item Geriatric Depression Scale (GDS15) and the development of short versions. Fam Pract. 1994;11(3):260-266.

19. de Craen AJ, Heeren TJ, Gussekloo J. Accuracy of the 15-item geriatric depression scale (GDS-15) in a community sample of the oldest old. Int J Geriatr Psychiatry. 2003;18(1):63-66.

20. Sugishita K, Sugishita M, Hemmi I, Asada T, Tanigawa T. A validity and reliability study of the Japanese version of the Geriatric Depression Scale 15 (GDS-15-J). Clin Gerontol. 2017;40(4):233240.

21. Sugishita M, Hemmi I, Takeuchi T. Reexamination of the validity and reliability of the Japanese version of the Mini-Mental State Examination (MMSE-J). Jpn J Cogn Neurosci. 2016;18:168-183.
22. Shimoda K, Kimura M, Yokota M, Okubo Y. Comparison of regional gray matter volume abnormalities in Alzheimers disease and late life depression with hippocampal atrophy using VSRAD analysis: a voxel-based morphometry study. Psychiatry Res. 2015; 232:71-75.

23. Hirata Y, Matsuda H, Nemoto K, et al. Voxel-based morphometry to discriminate early Alzheimer's disease from controls. Neurosci Lett. 2005;382:269-274.

24. Koyano W, Shibata H, Nakazato K, Haga H, Suyama Y. Measurement of competence: reliability and validity of the TMIG Index of Competence. Arch Gerontol Geriatr. 1991;13:103-116.

25. Saito M, Kondo N, Aida J, et al. Development of an instrument for community-level health related social capital among Japanese older people: The JAGES Project. J Epidemiol. 2017;27:221-227.

26. Zhang X, Dupre ME, Qiu L, Zhou W, Zhao Y, Gu D. Urban-rural differences in the association between access to healthcare and health outcomes among older adults in China. BMC Geriatr. 2017; 17(1):151.

27. Ishii S, Tanaka T, Akishita M, Ouchi Y, Tuji T, Iijima K; Kashiwa study investigators. Metabolic syndrome, sarcopenia and role of sex and age: cross-sectional analysis of Kashiwa cohort study. PLoS One. 2014;9:e112718.

28. National Institute for Longevity Sciences Longitudinal Study of Aging. Monograph: The first wave. http://www.ncgg.go.jp/cgss/ department/ep/monograph1st/index.html; 1997-2000. Accessed 15.04.2019. 